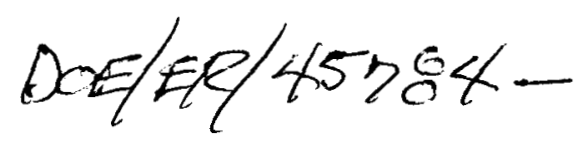

Progress Report for DOE Sponsored Research (DEFG02-99ER45784) on

\title{
Search for the Microscopic Origin of Defects and Shear Localization in Metallic Glasses
}

Program Duration: September 1999 - August 2002

\section{Sumitted to:}

Dr. Robert Gottschall \& Dr. Robert Kwang

Division of Materials Science, ER-13

U.S. Department of Energy

Office of Basic Energy Sciences

19901 Germantown Road, Germantown, MD 20874-1290

Principle Investigator:

\section{Mo Li}

\section{School of Materials Science and Engineering}

Georgia Institute of Technology

771 Ferst Street, N.W.

Atlanta, GA 30332-0245

Phone: 404 - 385 - 2472

Fax: 404 - 894 - 9140

Email:mo.li@mse.gatech.edu

URL: http:// www.mse.gatech.edu/people/faculty/li/li.html

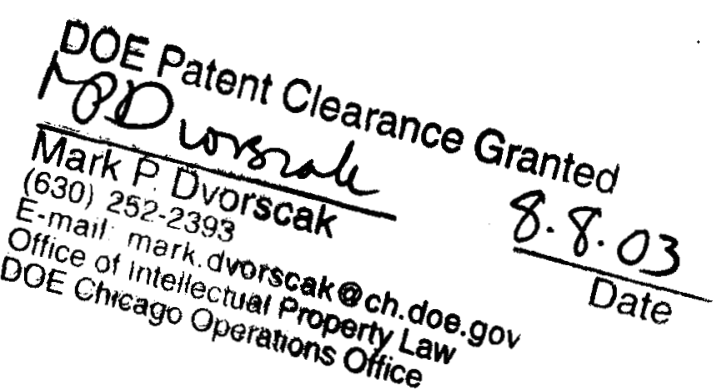




\section{DISCLAIMER}

This report was prepared as an account of work sponsored by an agency of the United States Government. Neither the United States Government nor any agency thereof, nor any of their employees, makes any warranty, express or implied, or assumes any legal liability or responsibility for the accuracy, completeness, or usefulness of any information, apparatus, product, or process disclosed, or represents that its use would not infringe privately owned rights. Reference herein to any specific commercial product, process, or service by trade name, trademark, manufacturer, or otherwise does not necessarily constitute or imply its endorsement, recommendation, or favoring by the United States Government or any agency thereof. The views and opinions of authors expressed herein do not necessarily state or reflect those of the United States Government or any agency thereof. 


\section{DISCLAIMER}

Portions of this document may be illegible in electronic image products. Images are produced from the best available original document. 


\section{CONTENTS}

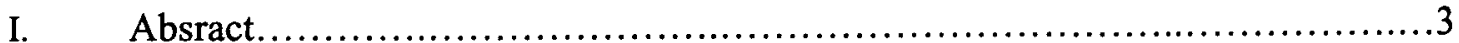

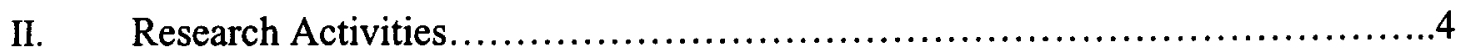

2.1 Search for Atomic Defects in Glasses -- Free Volume Identification

2.2 Effect of Disorder on Deformation and Fracture -- Disordered Ising Model

2.3 Mesoscopic Modeling -- Phase Field or Ginzburg Landau Theory

III. Impact and Summary of Major Accomplishments.......................... 12

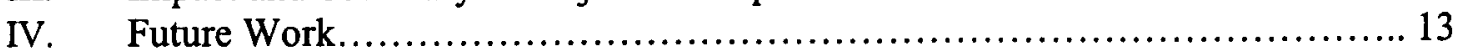

V. Personnel Support........................................................ 14

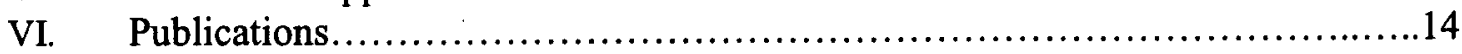




\begin{abstract}
This proposed research addresses one of the long outstanding fundamental problems in materials science, the mechanisms of deformation in amorphous metals. Due to the lack of long-range translational order, details of structural defects and their behaviors in metallic glasses have not been accessible in experiments. In addition, the small dimensions of the amorphous alloys made early by rapid quenching impose severe limit on many standard mechanical and microscopy testing. As a result, the microscopic mechanism of deformation in the amorphous materials has not been established. The recent success in synthesis of bulk metallic glass overcomes the difficulty in standard testing; but the barrier for understanding the defect process and microscopic mechanisms of deformation still remains.
\end{abstract}

Amorphous metals deform in a unique way by shear banding. As a result, there is no work hardening, little macroscopic plasticity, and catastrophic failure. To retain and improve the inherent high strength, large elastic strain, and high toughness in amorphous metals, a variety of synthesis activities are currently underway including making metallic glass matrix composites. These new explorations call for a quantitative understanding of deformation mechanisms in both the monolithic metallic glasses as well as their composites. The knowledge is expected to give insight and guide to design, processing and application of this new generation of engineering materials.

This DOE funded research takes the approach of computer simulation and modeling to tackle this problem. It is expected that with the increasing power of computers, the numerical modeling could provide the answers that are difficult or impossible to get from experiments. Three parallel research tasks were planned in this work. One is on search of atomic structural defects and other microscopic mechanisms underlying the deformation process. The second is to formulate a general model to describe shear localization, shear band formation and propagation on mesoscopic scale. The last is to determine the constitutive behaviors of the amorphous metals from the knowledge gathered from the atomistic and mesoscopic modeling, as well as experiments. The continuum description of deformation and fracture in metallic glass is expected for predicting and analyzing mechanical performance of bulk metallic glass products and components in real applications.

With the support of the DOE grant, several major breakthroughs have been made. Among the highlights are (1) quantitative characterization of free volumes, (2) dynamic modeling of breakdown process in disordered Ising models, and (3) development of a novel mesoscopic modeling method using phase field, or Ginzburg-Landau Theory. These progresses laid a firm foundation for the future advance in comprehensive understanding of deformation mechanisms in amorphous metals.

The future works are laid out that address not only the remaining or unfinished tasks and topics, but also the further extension and development from the knowledge learned from the current research. Among these topics are (1) micromechanics of defects, (2) composite modeling, (3) a theory of shear localization by combining microscopic defect properties with mechanics, and (4) continuum modeling of glassy metal composites and products in services. 


\section{RESEARCH ACTIVITIES}

Under applied stress, for example, tension or compression, metallic glass deforms by forming tiny bands, ranging in thickness from hundreds of nanometers to microns. The surface morphology of these bands, although larger in scale, resembles strikingly that of the slip bands in crystalline materials. The fundamental difference, however, between the shear band and the slip band is in the following four aspects: (1) Slip bands become delocalized, or spread after certain amount of slips occurring in one location. Shear bands, on the other hand, remain localized till either fracture occurs in the same location, or arrest when other larger shear bands form nearby. (2) The fracture surface shows vein pattern in amorphous metals, while crystals have either smooth surface in brittle fracture or rough surface in ductile fracture. (3) The crystal structure behind the moving dislocation is left intact; while the region behind the deformation front accumulate residual deformation in metallic glass. (4) There are entanglement and interactions between the cross slipping dislocations, but there is no interaction between two shear bands when they pass each other.

The deformation behaviors for crystalline materials can be understood with the knowledge of dislocations. The delocalization behavior of the slip band, for example, is caused by the interaction, or entanglement, of dislocations activated and moving on different crystal slip planes. The roughness of the fracture surface is caused by formation of either voids or dislocation networks. Understanding of shear banding in amorphous metals, however, encounters tremendous difficulties. The following questions basic to understanding shear banding are still open: What are the underlying defects that promote deformation? What are their responses and behaviors under applied stress? How does shear band form and propagate? And finally what governs the morphology, shear band thickness, and distance between two bands?

The early works provided some insights but most of these questions remain untouched. The foremost issue needed to be resolved is to identify and characterize defects underlying the deformation process. Free volume is the "defect" that was hypothesized being responsible for promoting deformation in metallic glass. However, unlike dislocation that is a well-characterized line defect, free volume is an ill-defined one. It can be thought as a low-density region involving many atoms, or a void associated with a particular atom. Since there is no long-range order in amorphous metals, the free volume, or any other structural defects in metallic glass, cannot be directly imaged by strain contrast through Bragg diffraction.

In this research, we performed extensive molecular dynamics simulation to identify and "map" out these defects in glasses. In the following section, we summarize the results in the current work.

\subsection{In Search of Atomic Defects in Glasses - Free Volume Identification}

In order for amorphous materials to deform, local "disorder" is needed (in the same fashion as dislocation is needed for deformation in crystalline materials). Homogeneous deformation involving a large number of atoms is energetically too costly to be possible. However, with the atoms randomly situated in metallic glass, it is hard to 
imagine what type of disorder should exist in this already highly disordered material, and furthermore, how would these defects, if exists at all, be responsible for deformation.

In order for atoms to move in amorphous metals, there must be some extra spaces distributed in metallic glass, so atoms can move, or deform locally, from one place to another. According to the free volume theory for the glass transition, the excess spaces in the glass-forming liquid decrease below some critical value at and below the glass transition temperature. Therefore, free volumes could exist in amorphous materials.

Our work involves two tasks: (1) Determine whether or not there are free volumes inherited from the glass-forming liquid after cooling, although the theory predicts a small and scattered ones. (2) Determine whether or not free volumes can also be created under the applied stress. (3) How the free volumes nucleate and form in the early stage of plastic deformation.

We used molecular dynamics to identify the possible free volume defects. The free volume is defined as the extra space surrounding each atom, $V_{f}=V_{i}-V_{0}$, where $V_{i}$ is the Voronoi volume for each atom $i$ and $V_{0}$ is the atomic volume (the hard-core volume). Following the definition of $V_{f}$ by Turnbull, only those spaces $V_{i}$ that are larger than a critical value (for example, beyond the volume determined by the radius of the reflection point in the interatomic interaction) are the volumes that can accept or promote displacement of atoms. These volumes are called free volume. The free volumes in both the glasses made from the rapid-quenched liquid and under applied stress are to be characterized.

Figure 1 shows the fraction of atoms that have free volumes. The free volume decreases as the sample cooled. As shown by this simulation, free volumes can indeed be trapped in the glass. In fact, faster cooling will trap more free volumes. The amount of free volume trapped in glass range from $10 \%$ to $18 \%$ within the cooling rates affordable in molecular dynamics simulations.

Besides the "intrinsic" free volumes inherent from the glass-forming liquid, free volumes could be generated during deformation. The free volume density changes under applied shear loading. Figure 2 is a snapshot of the atoms and the extra (free) volumes in a shear band. These results are obtained from the molecular dynamics simulations of glassy systems quenched from the melt. It is clear that shear band indeed forms with larger than the average of atomic volumes.

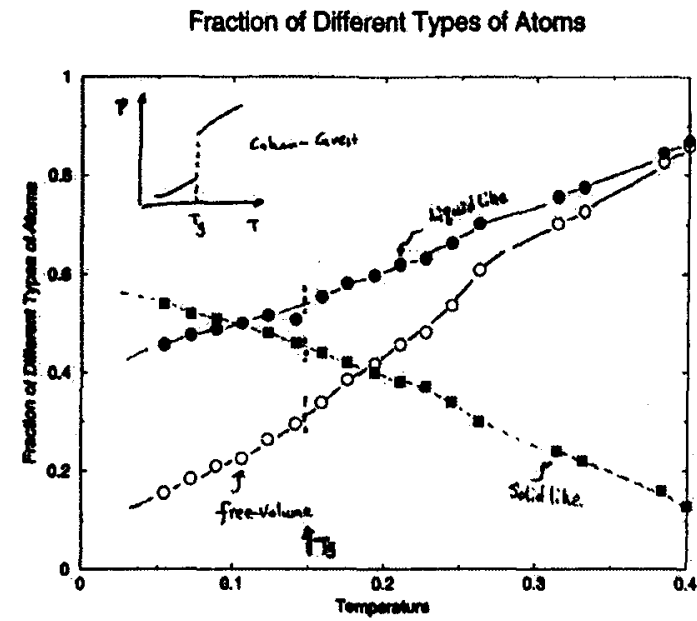

Figure 1. The fraction of atoms whose volumes are larger, or have free volumes. 
These findings confirm the long outstanding suspicion that some type of structural change should underlay the shear banding. In particular, the critical experiment done by Tumbull indicates that there are some permanent "damages" in the shear bands after deformation. He showed that the shear bands appear at the same location on the sample repeatedly bent; and only after annealing the new shear bands show up in different locations. Our works show that it is the free volumes in the shear band that remain inside after deformation; and they become the "seeds" or nuclei for further deformation.

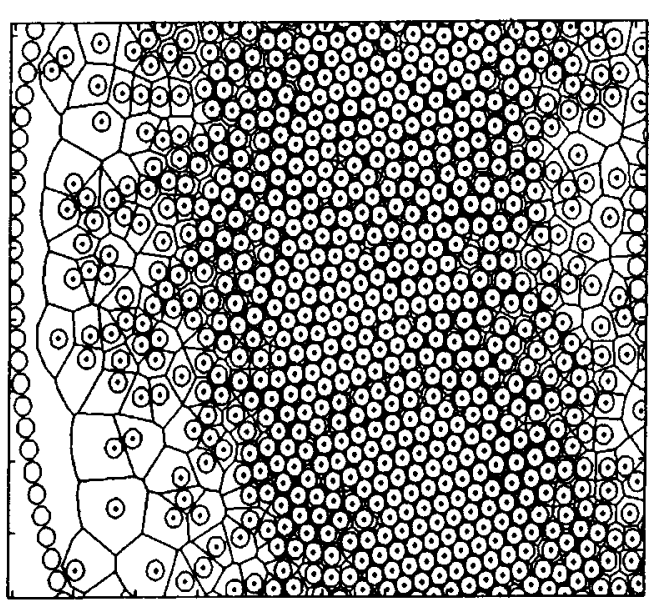

Figure 2. Atomic volumes (Voronoi volumes) inside and outside shear bands.

The extra space, or free volume, is needed in order for atoms to move. However, several key questions still need to be addressed: (1) From our simulations, the free volumes are not "void-like" defect. They are scattered and associated with the atoms with, very often, just slightly larger volumes. (2) Shear bands are made of atoms with "free volumes" or low densities. However, the deformation zones, or shear localization, do not form on every existing free volumes, even the size of some of these free volumes are quite large sometimes. Why does the shear localization happen selectively? (2) The extension or the thickness of shear band is rather small, and two bands can even move cross each other, indicating a lack of long-range interactions between the disordered/free volume defects. This is in sharp contrast to dislocations in crystalline materials. What is the physical reason for this short-range interaction, or non-interacting property? (3) How does the hydrostatic pressure alter the shear localization behavior given the observation that certain local volume dilatation is needed? These questions fundamental to understand shear banding will constitute the core of the future work.

\subsection{Effects of Disorder on Deformation and Fracture - Disordered Ising model}

Another critical aspect of the deformation process is related to how the deformation proceeds or propagates. As discussed above, the deformation in metallic glass proceeds through free volume formation. The deformed regions are permanently defected with certain residual free volumes. This phenomenon is in sharp contrast to the deformation in crystalline materials: When a dislocation swipes through a slip plane, no permanent defect or damage is left behind (except at the late stage when dislocation forest and networks form). Due to this fundamental difference, we ask how the deformation front between the deformed and undeformed region moves in metallic glass?

In crystalline materials, this deformation front is dislocation. We can imagine that the front of the shear band could also be a similar kind of defect dividing the undeformed and deformed regions. Due to the accumulated deformation behind the front, this defect is not the same as the crystal dislocation. Both Volterra and Somigliana dislocations are 
the dividing lines of deformed and undeformed regions. The former has a deformation zone with varying displacements and the later has uniform displacements. Our simulation results suggest that shear band resembles Voterra dislocations. However, there are many questions need to be clarified to fully characterize this defect. (1) What is the characteristic strain/stress field for this Volterra-like shear band front? (2) Why can they cross each other without interaction? (3) How does this "defect" propagate under applied stress? And finally, how does it interact with obstacles?

In this section, we will summarize some of our efforts in answering the last two questions: How shear band front propagates and how it interacts with obstacles. Answers to these questions have a practical importance since the current efforts to make bulk metallic glass composites requires us to have a detailed knowledge of how the deformation front could interact with various objects and inclusions. Since this deformation front resembles the dislocation in many ways, structural inhomogeneities, second phase particles, and other dislocations all affect its motion. In amorphous metals, such obstacles are numerous. It can be foreign objects embedded during making of composites, or it can be crystallites nucleated thermally or mechanically, or even the preexisting free volumes that are inherited from the glass-forming liquid. They all could influence the deformation front motion. The question that we need to ask is how these structural inhomogeneities in the glass affect the dynamics of the deformation front motion?

To answer these questions, we carried out Monte Carlo simulations. The model systems we used are Ising spins. The primary reason for us to use spins versus atoms is that we could handle a large system because each spin could represent a group of atoms. Therefore, the spin model is effectively a coarse-grained system. Although many differences remain between atoms and spins, how the deformation front propagates in these two systems is very similar: The breaking or shifting of the atomic bonds in the front of the shear band is analogous to the two Ising spin states, up or down. Also this breaking and rearranging of the bond costs energy and is irreversible; these features are simply represented by the flipping process of the Ising spins which interact with each other through the "coupling" constant, J, and their motions are governed by irreversible Glauber dynamics.

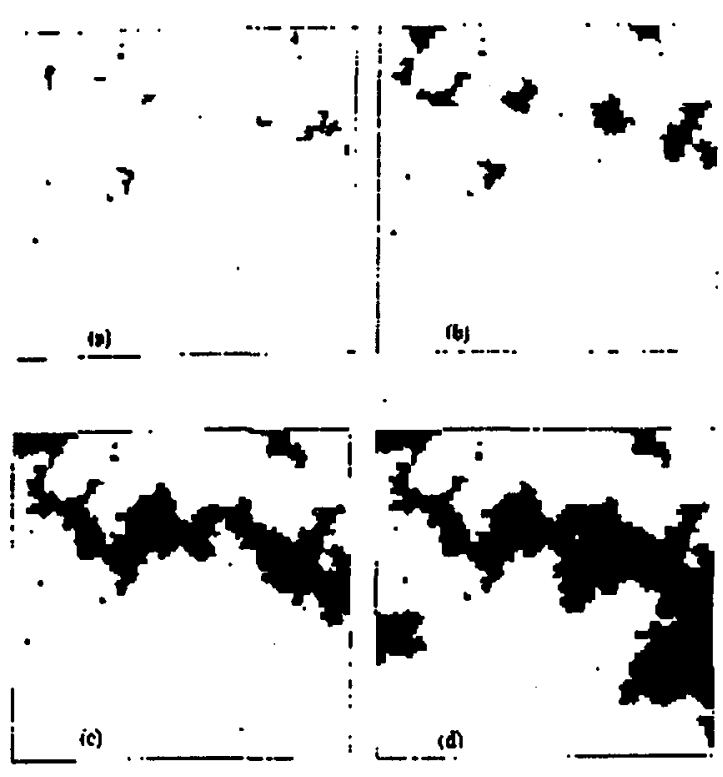

Figure 3. $\quad$ Flipped Ising spins under the applied field. (a)-(d) are a sequence of flipped spin avalanche formation, or "shear band" formation. 
Figure 3 shows deformed regions (in black areas) as represented by the flipped spins under an applied magnetic field. These regions can be considered as those of the free volumes and the magnetic field is analogous to the applied stress. It is clear to see in Figure 3 that the deformation occurs at some local regions with "free volumes", or flipped spins in the Ising model. However, same as in the MD simulation, the "shear band" does not form from every existing defected region. It forms collectively by combining different deformed regions, some of which are the pre-existing ones and some are newly created.

In addition, we can obtain a quantitative measurement of how the deformation front moves, or how the deformation regions grow. As shown in Figure 4, the growth of the deformed regions follows two types of dynamic process, which are characterized by two dynamic scaling laws. One is a slow "nucleation" process in which small and scattered deformations zones form under applied field; and the other one is the faster "growth" process at the later stage in which deformation regions emerge and coalesce. The early nucleation of the small deformation zones are independent of each other and the "obstacles"; they show a universal behavior in dynamic behavior regardless of the different environments and even the variations in the coupling/interactions between spins. At the later stage when the deformed zones are larger, the variation in both the environments and interactions starts to show influence. The deformation front can be pinned by the obstacles, but the coalescence between different deformation zones becomes dominant. Therefore, the deformation is faster

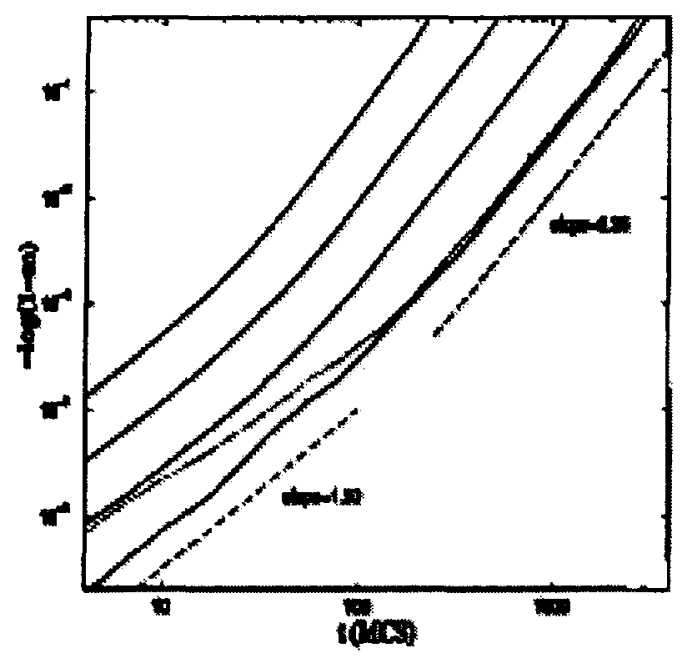
(Fig. 4).

Figure 4. The number of flipped spins versus time. Two different growth regimes are seen.

These findings shed a great deal of light to our understanding of shear band front propagation. We learned from the above properties that shear band front is quite different from dislocation motion and dislocation mediated deformation: For example, dislocation can annihilate each other; but shear zones in amorphous materials cannot. The speed of a single moving dislocation does not vary drastically under a constant applied stress and without the presence of other dislocations. But the speed of a single shear band front has two distinct regimes.

It should be mentioned that two types of Ising models are used in our simulations. One is the random field Ising model where pre-existing random field is included in the model to liken the pre-existing defects, cracks, or other free volumes. The second is the random bond Ising model where varying interactions between the spins are used to mimic the different atomic densities or interatomic interactions in regions with varying density. Our Monte Carlo simulation gives the same dynamic behavior for the deformation 
process for both models, indicating that the type of disorder, free volumes, foreign obstacles, or crystalline precipitates should not alter the general dynamic behaviors of shear bands.

\subsection{Mesoscopic Modeling - Phase Field Method or Ginzburg Landau Theory}

The atomistic MD and Monte Carlo simulations provided, for the first time, details of the microscopic deformation process. We not only learned that deformation and shear band formation are direct manifestations of free volumes, but also how the shear band nucleates and propagates and how deformation interacts with inhomogeneities. The next step in our tasks is to formulate a mesoscopic model for shear band. The primary motivation is that we need this model to include the information of defects as well as the dynamics of shear band formation and propagation on a larger scale. (The thickness of shear bands is typically of the order of microns.) Both MD and Monte Carlo simulations are too small in scale to accomplish this task. The continuum model such as the finite element method (FEM), although able to cover large scales, is too crude to be able to include the detailed defect properties. Besides, we do not have the input constitutive properties of metallic glasses necessary for carrying out the FEM modeling.

We formulated a novel approach, based on the Ginzburg-Landau theory of phase transition, for the mesoscopic modeling of shear band to include free volumes and their dynamic behaviors. The insights for this formulation are provided partially by both the $\mathrm{MD}$ and Monte Carlo simulations. The general formulation of this method was described early in the DOE proposal. In the following, we present a brief summary of this model.

The basic idea is that the deformation process, as seen from the MD and $\mathrm{MC}$ modeling, resembles a phase transition, where the new phase is the deformed region and the parent/old phase is the undeformed region. This assumption is valid as suggested by the properties and behaviors of the shear zones in amorphous materials seen from our simulations. It is also interesting to notice that the origin of this approach can be traced back to Griffith. The Griffith theory of fracture can be reformulated into the theory of nucleation for phase transitions where the competing process between the newly created fracture surfaces and the elastic energy in the body determines the onset of fracture and the crack length.

For amorphous metals, the free energy function for the system containing free volume defects has three parts,

$$
F=F_{0}+\frac{1}{2} \Sigma_{i j k l} c_{i k l} \varepsilon_{i j} \varepsilon_{k l}+\int_{v}\left[A \rho^{2}(r)+B \rho^{3}(r)+C \rho^{4}(r)+\frac{1}{2} \kappa|\nabla \rho(r)|^{2}\right] d r
$$

where the first term is the free energy of the system due to the presence of the free volumes. The second part is the elastic energy due to the applied external stress on both deformed/free-volume regions and undeformed regions. And the last part is the interface energy between the deformed and undeformed regions, or of the deformation front. It is an extension from the Griffith theory that the deformation or shear zones contribute to the first term. As a contrast, it is the fracture surface energy that is used in Griffith theory. In this work, we have the "interface" which has a far broader sense. The interface covers the 
situations raging from the boundaries between the deformed and undeformed zones where the free volume density is small to fracture surface where the free volume density is large. This formulation is only possible for shear banding since complete decohesion between atoms do not occur as in fracture; we are describing a gradual change in "defect" density, the free volumes.

The deformation process is described by the free volumes; If the free volume density $\rho$ is zero, there is no deformation; if the free volume reaches one, the deformation becomes a fracture where debonding occurs. Here $\rho=V_{f} / V_{f}^{\max }=\left(V_{i}-V_{0}\right) /\left(V_{i}^{\max }-V_{0}\right)$, where $V_{f}^{\max }=V_{i}^{\max }-V_{0}$ is the maximum free volume at which decohesion or debonding occurs; $V_{f}^{\max }=R_{C}{ }^{3} V_{0}$ and $R_{C}$ is the cut-off distance of the interatomic interaction. When $V_{f} \rightarrow$ $V_{f}^{\max }$, or $\rho \rightarrow 1$, the atoms are separated by the distance equal or larger than $R_{C}$, fracture occurs. At $0<p<1$, the free volume manifests as shear band, or deformation zone since no decohesion occurs.

The dynamic equation that governs the evolution of the free volume also describes the deformation and fracture process. We can write this equation from the free energy function as

$$
\frac{\partial \rho}{\partial t}=-\Gamma \frac{\partial F}{\partial \rho}+\xi
$$

where $\Gamma$ and $\xi$ are the relaxation time and the thermal fluctuations at the temperature $T$ respectively.

In the following, we present two case studies using this new method. One is the deformation in a homogeneous and infinitely large amorphous sample under an applied tension. Another is the testing of a mode I crack.

Figure 5 shows the shear band formation and the final fracture surface morphology. Clearly the free volume evolves from isolated defects in the sample into a local stripe like bands. The free volume evolution resembles strikingly the free volume changes from our MD simulations showed early. However, due to the small sample size in $\mathrm{MD}$, the vein pattern cannot be seen using the atomistic modeling. The mesoscopic simulation reveals that the vein pattern forms at the regions with high free volume densities. The instability of the free volume regions occurs when the applied stress reaches the yield point. The deformation starts propagates; but the free volume regions in the front of the propagating deformation zone is short-range. This result indicates that the deformation strain field in amorphous metals, as described by the changing free volumes, is very narrow and very localized. As a contrast, the strain field of dislocations is long-range in crystalline materials. This localized strain field is likely the reason why shear band are not interacting and can cross-slip easily. The lack of work hardening in amorphous metals can also be explained by this unique property of the shear band.

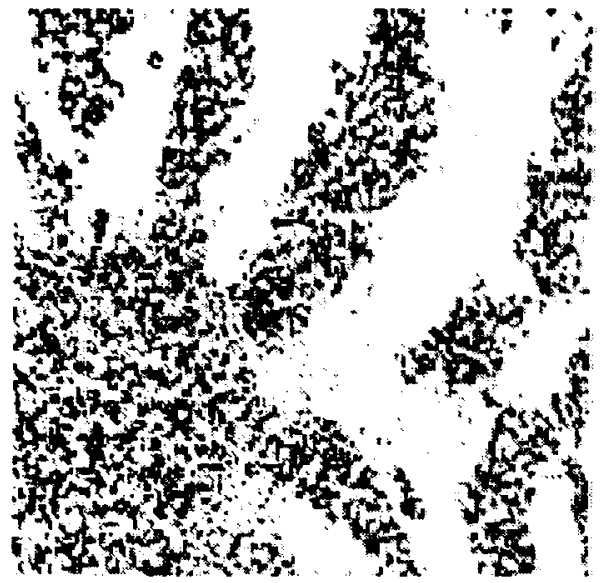

Figure 5. Vein pattern. 
In addition, this mesoscopic, as well as the atomistic modeling, show that the shear bands form from local free volume zones that merge in a coherent fashion. Also both the thickness of each band and the distance between bands are quite well modulated. These free volume density "waves" $\rho(r, t)$ are influenced by the following properties: (1) If the interface energy is high, the bands are narrow; if the interface energy is low, the band is wide or thick. The elastic modulus of the amorphous materials also influences the band thickness. The higher it is, the narrow the band. The narrow band forms when the strain rate is high. (2) The inter-band distance is the results of the late stage free volume density modulation. It is less influenced by the original free volume density distribution, or other impurities/obstacles. It is largely related to the internal stress distribution or relaxation of the system under the applied stress: When many deformation zones form, the small ones will disappear when a bigger one appears which reaches the local yield/instability condition. Each of the surviving bands will carry with it the smaller internal stress. This self-regulating process ultimately leads to the regular shear band pattern.

Figure 6 shows the shear band formation and fracture in a single notch configuration. This test is a standard one also in experiments. Here we summarize several important results. The branching or shear band multiplication can be seen clearly in the regions in the front of the crack tip where free volumes are generated. Figure 7 shows the crack velocity. When branching occurs, the crack velocity starts to oscillate.

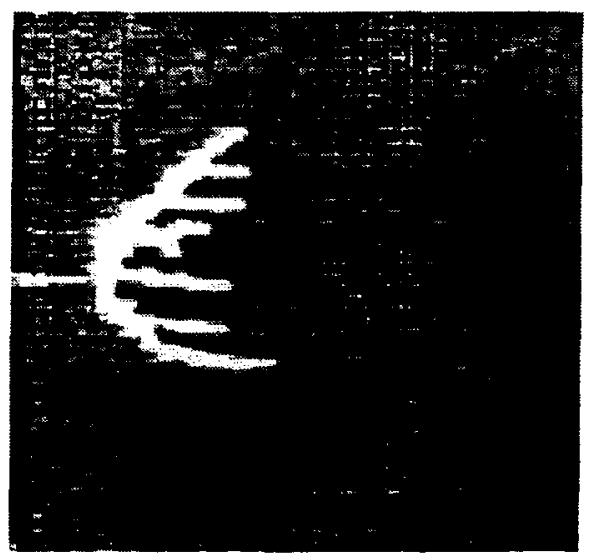

Figure 6. Branching of the opening of shear bands from a Mode I fracture.

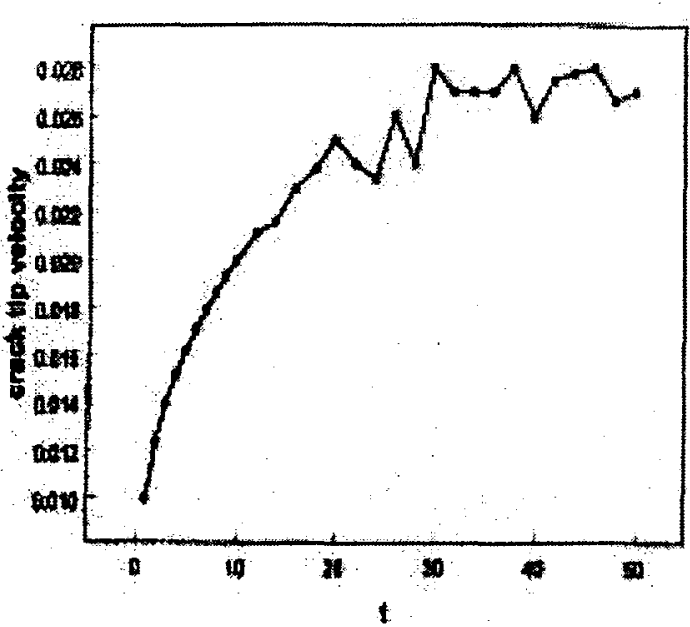

Figure 7. Crack velocity. 


\section{ACCOMPLISHMENTS AND IMPACTS}

In the past two years, we have accomplished several major tasks that paved the way toward the goal of a full and quantitative understanding of the microscopic mechanisms of deformation in metallic glass.

1. In the atomistic modeling, we developed a systematic method to characterize free volumes; and for the first time, we established the direct link between the free volumes and shear band formation.

2. We also performed Monte Carlo simulations of the Ising models from which the dynamic behavior of deformation is established. Two-stage of dynamics evolution of "shear bands" is identified. One is the nucleation of the shear zones and the second is the propagation of the deformed zones. We also demonstrated that in the nucleation stage, the effect of different disorder and inhomogeneities on the dynamics of deformation does not alter the general dynamic deformation behavior; and they play an important role only in the later "growth" stage.

Another critical result from this study is the revelation of the nature of the shear band. It is a band with continuous distribution of deformation inside, which is different from crystal dislocations.

3. We developed a novel mesoscopic model from the Ginzburg-Landau theory for phase transitions. This new field model can simulate deformation and fracture on mesoscopic scale by combining microscopic flow defects and the dynamics evolution of deformation on a larger scale.

The pattern formation of the shear bands can be described by the free volume density wave $\rho(r, t)$. The band thickness is affected by intrinsic as well as extrinsic parameters such as the interface energy, elastic modulus and strain rates. The interband distance is controlled by stress/strain relaxation and instability of deformation zones.

In addition to the insights we gained from this work, several new methods are developed. These new methods allow us to continue the planned research in metallic glasses. Moreover, they also provide novel and effective approaches for other communities sharing the same fundamental problems in shear localization. For instance, we recently applied the free volume characterization technique successfully to sand and other granular materials that have shear localization. The dynamics scaling in our Monte Carlo simulation provided a very efficient way to obtain the critical exponents that otherwise have to be obtained from equilibrium scaling which is tedious and often inaccurate. The phase field model can also be used in other material systems such as polymers, granular materials, and metallic glass composites. 


\section{FUTURE WORK}

It is evident that the efforts and accomplishment made in the last two years are just a beginning. Our efforts so far have been focused mainly on development of various methodology and algorithms. With the initial success in methodology development, we are now able to pay more attentions toward the in-depth study of shear banding and shear localizations. In the following, we list several main research thrusts that are a natural extension of the current work. The details of the proposed future work can be found in the proposal.

\section{(1) Continuation:}

We shall focus on three-dimensional samples with realistic interactions. So comparison with experiments can be made.

For free volumes, we shall continue to push the limit in including mode atoms till more than one shear band can be seen. With this, we could apply our characterization tools to identify and measure free volumes.

\section{(2) Micromechanics of Free Volume and Shear Band Front:}

Considerable amount of effort will be devoted to identifying and measuring stress and strain field around the shear front and shear band. We shall use both simulations and analytical theory to tackle this problem. The purpose for this is (1) we need to characterize the nature of the shear band and shear front to see if they are different from dislocations; (2) for modeling of composites, it is necessary to have a quantitative answer to the stress and strain distribution for shear band when interacting with obstacles.

\section{(3) Adiabatic Heating and Heat dissipation:}

We will continue our effort to develop algorithms to handle heat dissipation and production in metallic systems. MD and other techniques are apparently not adequate to deal with electron-assisted heat conduction, nor with heat conduction itself computationally. We will implement and test two algorithms we came up early. Ultimately we will use these tools in modeling adiabatic heat in shear banding.

\section{(3) Modeling of Metallic-Glass-Matrix Composites:}

The knowledge we learned from our previous work and the methodology allow us study various issues in composites. We will first apply the mesocopic method to model inclusion effects of deformation (shear banding, strength and touchness change). The advantage of this technique is that we do not need to know the constitutive laws as in continuum modeling. By solving the boundary problems for inclusions in the metallic glass matrix, we can model the deformation and fracture process.

\section{(4) Continuum Mechanics and Modeling and Constitutive Relations:}


We will continue our quest for various constitutive behaviors of both monolithic and composite metallic glasses. We will take the combined methods, using $\mathrm{MD}, \mathrm{MC}$ and mesoscopic methods, along with experiment input, to establish the relation of stress and strain, strain rate, temperature, structural and chemical heterogeneity, and stress and strain field.

\section{PERSONELL}

The activities presented above are supported by the DOE grant. It started in September 1999. Three people, the PI, a graduate student, and a postdoctoral fellow have been involved in this research full time. The PI is mainly responsible for development of simulation methods in atomistic modeling of free volumes and the glass transition. The graduate student is working with the PI on the Monte Carlo simulations of Ising models and implementation of the mesoscopic model. The postdoctoral fellow just joined the research group in September 2001. He is working on MD modeling of deformation in hard sphere models and other glassy systems. A second postdoctoral fellow, who will be partially supported with the DOE grant and PI's start-up fund, is expected to join the research starting in December 2001. His main responsibility is to develop analytical theory and continuum modeling for shear localization in continuum mechanics. The personnel with full and partial DOE support is listed below:

\section{Graduate Students:}

Guang-Ping Zheng: August 1998 - November 2001 (Ph.D, November 2001)

Xian-Ming Bai: $\quad$ August 1999 - present (Ph.D candidate, passed qualification)

Postdoctoral Fellows:

Payman Jalali: $\quad$ September 2001 - present

Guang-Ping Zheng: December 2001 - November 2002

Lan-Hong Dai: November 2001 - October 2002

Undergraduates:

Alex Donohue: June 2001 - September 2001 (summer research)

\section{PUBLICATIONS}

\section{Journal Publications:}

1. Zheng, G-P. and M. Li, "Short-Time Dynamics of an Ising Model on Fractal Structure", Phys. Rev. E, 62, 6253, 2000.

2. Li, M., "Defect-Induced Topological Order-to-Disorder Transition in TwoDimensional Binary Substitutional Alloys: A Molecular Dynamics Study", Phy. Rev. B, 62, 13979, 2000.

3. Zheng, G-P. and M. Li, "Dynamic Scaling of Avalanches in Disordered System", Phys. Rev. E, 63, 36122, 2001. 
4. Zheng, G.P. and M. Li, "Dynamic Scaling of Barkhausen Effects in Magnetic Glasses", Physical Revies B., accepted, November 2001.

5. Zheng, G.P. and M. Li, "Effects of Disorder on Critical Short-time Dynamics", Physical Review E, accepted, November, 2001.

6. Li, M, "Effects of Random Strain Field on First Order Phase Transition", Physical Revies B., accepted, November 2001.

7. Gross, D. and M. Li, "Construction of Microstructures for Poly- and Nano-Crystalline Materials for Numerical Simulation and Modeling", Journal of Applied Physics Letters, accepted, November 2001.

8. Zheng, G.P. and M. Li, "Ginzburg-Landau Theory of Deformation and Fracture of Glassy Materials”, submitted to Physics Review Letters, November 2001.

9. Zheng, G.P. and M. Li, "Free Volume, Deformation and Fracture in Glasses: A Mesoscopic Simulation", submitted to Physics Review B, November 2001.

10. Li, M., "Cluster, Percolation and Glass Transition in Lennard-Jones Liquids", submitted to Physics Review Letters, November 2001.

11. Bai, X-M and M. Li, "The Nature of Crystal Melting at Superheating", submitted to Physics Review Letters, November 2001.

12. Li, M. and D. Gross, "Quantitative Methods for Construction of Digital Microstructures for Poly- and Nano-crystalline Material Modeling and Simulations", in preparation, International Journal of Plasticity, 2002.

\section{Conference Presentations and Invited Talks:}

1. Li, M., "Cluster, Percolation and Glass Transition", MRS Fall Meeting, Boston, 1999.

2. Zheng, G.P., J. X. Zhang and M. Li, "Scaling and Universality of First Order Phase Transitions, APS March Meeting, 1999.

3. Li, M., "Density Inhomogeneity and Percolation Transition in Glass-Forming Liquids", APS March Meeting, 1999.

4. Zheng, G.P. and M. Li, "Kinetics of Phase Separation in Asymmetric Ternary Systems", APS March Meeting, 2000.

5. Zheng, G.P. and M. Li, Short-time Dynamics of Dilute Ising System", APS March Meeting, 2000.

6. Gross, D. and M. Li, "A New Algorithm for Hard Sphere Molecular Dynamics", APS March Meeting, 2000.

7. Li, M., "Growing Length Scale and Shear Rigidity in a Glass-forming Liquids", ", APS March Meeting, 2000.

8. Li, M, "Future Trend in Amorphous and Nanocrystalline Materials", (Invited) ARO Workshop, Challotsville, VA, Nov. 2000. 
9. Li, M., "Search for the Nature of The Glass Transition", (Invited) Department of Physics, Clark University, Feb. 2001.

10. Li, M., "Computational Materials Science: New Challenges and Opportunities", (Invited) Department of Materials Science and Engineering, Georgia Institute of Technology, Feb. 2001.

11. Li, M., "Cluster Lifetime and Heterogeneity in a Glass-forming Liquids", APS March Meeting, 2001.

12. Zheng, G.P. and M. Li, "Dynamic Inhomogeneity of Domains in Magnetic Glasses", APS March Meeting, 2001.

13. Li, M., "Search for the Nature of The Glass Transition", (Invited) Department of Physics, The Johns Hopkins University, April 2001.

14. Li, M., "Glass Formability: Topology, Cluster and Percolation", (Invited) Air Force Research Laboratory, April 2001.

15. Gross, D. and M. Li, "Reconstruction of Microstructures for Poly- and Nancrystalline Materials", Engineering Foundation Conference, June 4-9, Barga, Italy, 2001.

16. Li, M., "Search for the Nature of The Glass Transition", (Invited) Southeast Regional APS Meeting, Nov. 2001.

17. Li, M., "Glass Formability", (Invited) Department of Materials Science and Engineering, University of Virginia, Nov. 2001.

18. Li, M., "Effects of Defects and Random Strains on First Order Phase Transitions", MRS Fall Meeting, 2001.

19. Li, M, "Atomistic and Mesoscopic Modeling of Deformation and Fracture in Noncrystalline Materials", (Invited) School of Mechanical Engineering Department, Georgia Institute of Technology, Dec. 5, 2001.

20. Li, M, "Quantitative Methods for Simulation and Modeling of Nano- and Polycrystalline Materials", (Invited) $28^{\text {th }}$ International Conference of Plasticity, January 2002, Aruba.

21. Li, M, "Phase Field Modeling of Deformation and Fracture in Non-crystalline Materials", (Invited) TMS Annual Meeting, 2002.

22. Zheng, G. P. and M. Li, "Mesoscopic Modeling of Deformation and Shear Localization in Metallic Glass", TMS Annual Meeting, 2002.

23. Li, M, "Shear Banding and Shear Localization in Metallic Glasses", (Invited) The $2^{\text {nd }}$ United Engineering Foundation Conference on Bulk Metallic Glass, March 22-26, 2002, Taiwan.

\section{Ph.D and MS Thesis:}

1. Zheng, G. P., "Computational Modeling and Simulation of Dynamic Fracture and Breakdown Processes in Disordered and Amorphous Materials", Ph.D, The Johns Hopkins University, November 12, 2001.

2. X. M. Bai, "The Nature of Crystal Melting at Superheating", Master of Science, The Johns Hopkins University, December 2001. 